\title{
Sciatica in a Five-Year-Old Boy
}

\author{
Farzad Omidi-Kashani ${ }^{1}$, Ebrahim Ghayem Hasankhani ${ }^{1}$, Ehsan Rafeemanesh ${ }^{2}$ \\ ${ }^{I}$ Orthopedic Department, Orthopedic Research Center, Imam Reza Hospital, Mashhad University of Medical Sciences, Mashhad, Iran \\ ${ }^{2}$ Occupational Medicine Department, Faculty of Medicine, Mashhad University of Medical Sciences, Mashhad, Iran
}

\begin{abstract}
The prevalence of back pain during childhood is 50\%; in 22\% of these cases, a specific diagnosis can be found. Osteoid osteoma is a rare benign tumor that occursonly in $20 \%$ of cases and involves the spine. The aim of this report is to explain an atypical case of lumbar osteoid osteoma with significant neurologic deficit at a very young age. A five-year-old boy was presented with refractory pain in the low back and left extremity for approximately one year. Positive clinical findings were antalgic gait, stiff lumbar spine, weak left big toe extension force and a positive straight leg rising test on the left side. Paraclinical studies revealed osteoid osteoma in the left-sided pedicle of the fifth lumbar vertebra. With surgical excision, he recovered immediately. Lumbar osteoid osteoma should be suspected as the cause of low back pain or sciatalgia in any young patient.
\end{abstract}

Keywords: Osteoid osteoma; Children; Lumbar spine; Surgery

\section{Introduction}

Studies indicate that the prevalence of back pain in children have increased up to $50 \%$; a specific diagnosis can be found in only $22 \%$ of these cases [1]. A meticulous and cautiously performed history and physical examination offer physicians with a good database to track the appropriate imaging studies and laboratory assessment.

In children under the age of 10 years, the most common causes of back pain are discitis/osteomyelitis and spine tumors [1]. Osteoid osteoma is a rare benign tumor that occurs only in $20 \%$ of cases and involves the spine, mostly in the posterior elements of the lumbar vertebrae [2]. It has been reported that in $74 \%$ and $89 \%$ of patients, associated spinal deformity and marked spinal stiffness are also present, respectively [3].

Spinal osteoid osteoma is usually presented with no neurologic deficit in an adolescent boy aged 10 to 20 [3]. The aim of this report is to explain an atypical case of lumbar osteoid osteoma with significant neurologic defi$c i t$ at a very young age.

\section{Case Report}

A five-year-old boy was presented to our clinic with refractory pain in the low back and left extremity for approximately one year. There was no history of precedent trauma or underlying disease. The parents declared that they repeatedly observed the patient awaken from sleep due to pain although it was not severe enough to hinder his play with peers. Within a year, the pain did not get much worse or better. The mother quoted that she was sometimes obliged to use ibuprofen to relieve the pain, and it was completely effective.

On physical examination, the child suffered from antalgic gait. His pain was mainly located around the lower back of the trunk and left lower limb and aggravated by coughing. He was not able to flex his lumbar

Received Jan 9, 2013; Revised Jul 4, 2013; Accepted Jul 16, 2013

Corresponding author: Farzad Omidi-Kashani

Orthopedic Research Center, Orthopedic Department, Imam Reza Hospital, Imam Reza Square,

Mashhad University of Medical Sciences, Mashhad, Iran

Tel: +98-915-514-9248; Fax: +98-511-859-5023, E-mail: omidif@mums.ac.ir 
spine. The range of motion of the left hip and knee joints was normal, but straight leg rising was severely limited. There was a mild tenderness without any swelling on the lumbosacral area. Deep tendon reflexes were completely intact, yet, the strength of the left big toe extensor was grade 2, according to the medical research council scale for muscle strength [4]. The boy did not complain of any remarkable associated paresthesia in his extremities.

There were no significant abnormalities in the laboratory studies. Due to the sciatalgic presentation, magnetic resonance imaging scan was undertaken. However, intervertebral discs and vertebral bodies had normal signal intensity and shape, and this modality did not help much. The first radiographs were reported as normal. On closer examination of recent radiographs, it was found that the left pedicle of the fifth lumbar vertebra has probably lost its normal elliptical hollow shape (Fig. 1). There was no evidence of spinal deformity as well.

Because we suspected a bony lesion, technetium-99m bone scan was requested. It showed an increased uptake in this area; ultimately, computerized tomography exactly localized the location of the lesion (Fig. 2).

With the initial diagnosis of a benign latent lesion (probably osteoid osteoma, acute on chronic unilateral spondylolysis, or some kind of infection such as Brodie's abscess), surgical resection was discussed and offered to the parents and the boy himself. They accepted and his father assigned the informed consent. Early after surgery, the pain completely disappeared and he was ambulated and discharged one and two days later, respectively. Histologic examination revealed characteristic nidus (contained immature osteoid with osteoblastic rimming) and reactive bone in the region of the sclerotic border compatible with osteoid osteoma. At the 3 year follow-up postoperatively, the patient did not have any significant problem and is able to perform all his daily activities, although he complains of occasional trivial low back pain.

\section{Discussion}

Low back pain in children and adolescents is more commonly caused by a serious problem relative to adults. Pri-
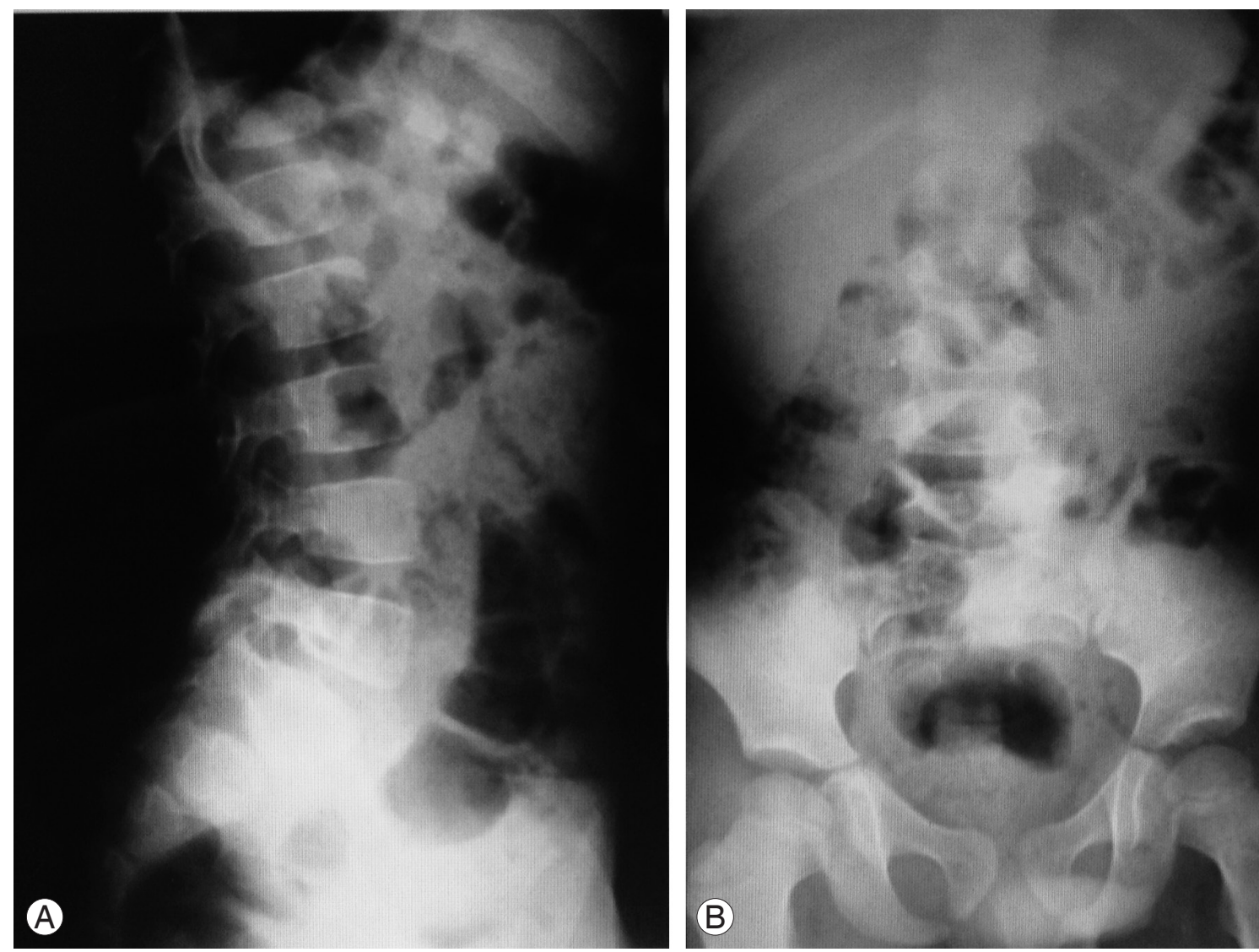

Fig. 1. (A, B) Anteroposterior and lateral lumbosacral views of the patient. 
mary osseous tumors of the lumbar spine are occasional in children; the most frequent ones are Ewing sarcoma, aneurysmal bone cyst, osteoblastoma, osteoid osteoma and primary lymphoma [1].

Although the clinical presentation of the patient which we reported was similar to that of patients with lumbar disc herniation, this entity has been accounted as being exceptionally below the age of 10 years and the magnetic resonance imaging scan did not confirm it [5].

In osteoid osteoma, usually long after the onset of clinical complains, radiologic studies remain normal. Therefore, in these settings, clinical suspicion of osteoid osteoma should be high and should be verified with a technetium bone scan [6]. So far, a negative bone scanning, patient with osteoid osteoma has never been reported [3].

The ordinary age of affection is between 10 to 20 years. Neurological deficit is usually uncommon with osteoid osteoma, although associated scoliosis and spinal stiffness are frequently reported [3]. In the case we presented, significant neurologic deficit accompanying with lumbar stiffness was observed. Due to atypical age and symptoms, there was a considerable diagnostic delay (12 months) in our patient, although it has been seen in other cases; a diagnostic delay of 5 years has been reported by Kaweblum et al. [7].

After diagnosis, surgical excision is usually recom-
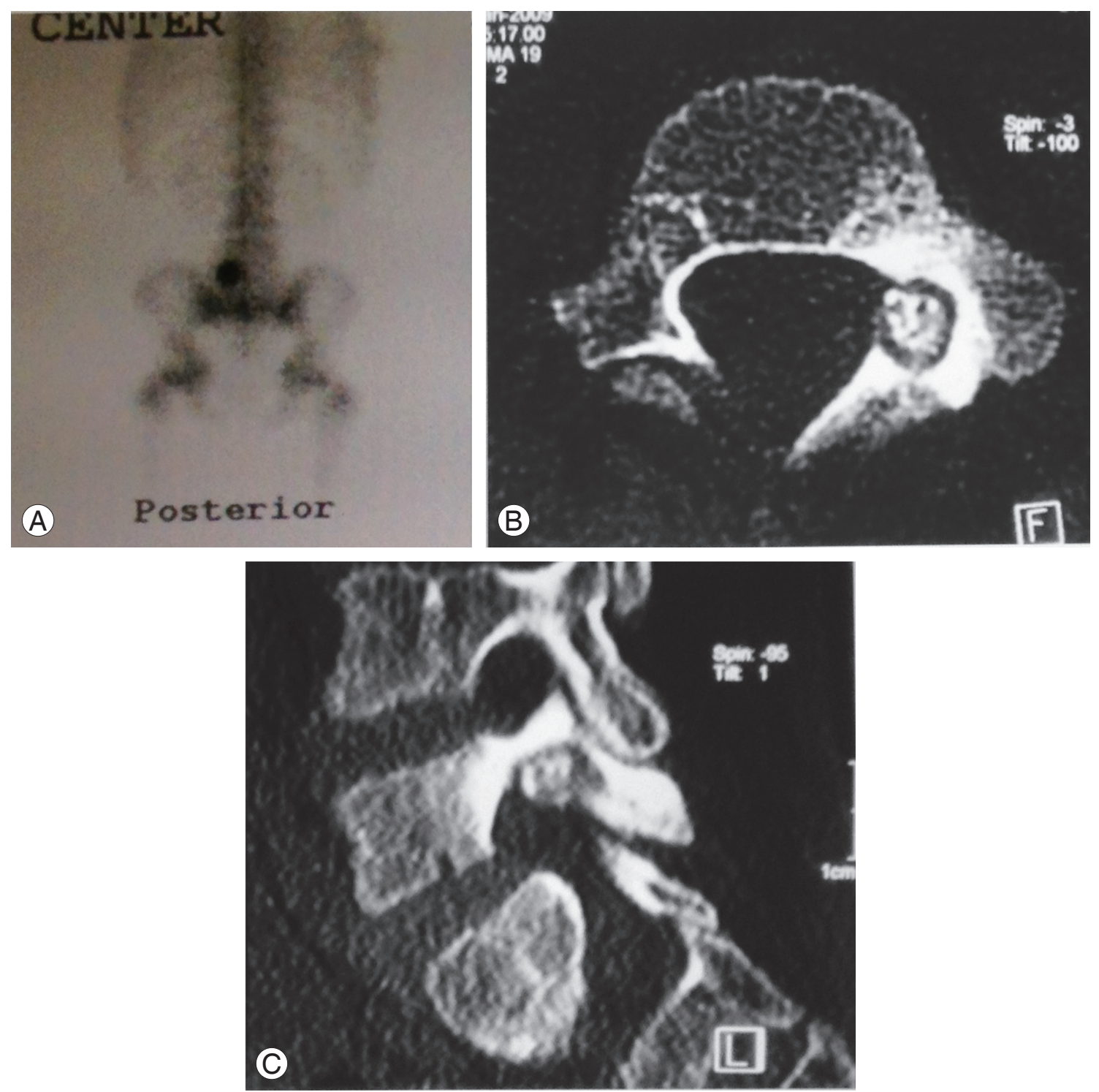

Fig. 2. Bone scan (A) showed increased uptake in left-sided pedicle of fifth vertebra;computerized tomography in axial and sagittal views $(\mathbf{B}, \mathbf{C})$ delineated the exact location of the lesion that was inferomedial to this pedicle. 
mended and after that, the pain is alleviated in a short time, as in our patient [5]. If the pain persists, it usually suggests incomplete excision. In conclusion, lumbar osteoid osteoma should be suspected as the cause of low back pain or sciatalgia in any young patient.

\section{Conflict of Interest}

No potential conflict of interest relevant to this article was reported.

\section{References}

1. Afshani E, Kuhn JP. Common causes of low back pain in children. Radiographics 1991;11:269-91.

2. Zileli M, Cagli S, Basdemir G, Ersahin Y. Osteoid osteomas and osteoblastomas of the spine. Neurosurg Focus 2003;15:E5.
3. Crist BD, Lenke LG, Lewis S. Osteoid osteoma of the lumbar spine. A case report highlighting a novel reconstruction technique. J Bone Joint Surg Am 2005; 87:414-8.

4. Medical Research Council. Aids to examination of the peripheral nervous system. Memorandum no. 45. London: Her Majesty's Stationery Office; 1981.

5. Martinez-Lage JF, Fernandez Cornejo V, Lopez F, Poza M. Lumbar disc herniation in early childhood: case report and literature review. Childs Nerv Syst 2003;19:258-60.

6. Feldman DS, Hedden DM, Wright JG. The use of bone scan to investigate back pain in children and adolescents. J Pediatr Orthop 2000;20:790-5.

7. Kaweblum M, Lehman WB, Bash J, Strongwater A, Grant AD. Osteoid osteoma under the age of five years. The difficulty of diagnosis. Clin Orthop Relat Res 1993;(296):218-24. 